\title{
Smart Glove Deployment for End-to-End Experimenting Palmar Pressure Assessments using Wearable IoT Technologies
}

\author{
Halysson C. S. Junior ${ }^{1}$, Artur F. da S. Veloso ${ }^{2}$, José V. V. Sobral ${ }^{3,4}$, \\ Ricardo A. L. Rabêlo ${ }^{2}$, Joel J. P. C. Rodrigues ${ }^{2,3,5}$, Antônio A. Rodrigues ${ }^{2}$ \\ ${ }^{1}$ State University of Piauí (UESPI), Teresina - PI, Brazil \\ ${ }^{2}$ Federal University of Piauí (UFPI), Teresina - PI, Brazil \\ ${ }^{3}$ Instituto de Telecomunicações, Universidade da Beira Interior, Covilhã, Portugal \\ ${ }^{4}$ Federal Institute of Maranhão (IFMA), São Luís - MA, Brazil \\ ${ }^{5}$ National Institute of Telecommunications (Inatel), Santa Rita do Sapucaí - MG, Brazil \\ \{halysson1007, arturfdasveloso\}@gmail, jose.sobral@it.ubi.pt, \\ ricardoalr@ufpi.edu.br, joeljr@ieee.org, junioraraujo03@gmail
}

\begin{abstract}
In the functional evaluations (FE), the lack of technological equipment for end-to-end hand grip experiments create difficulties in offering feedback to physiotherapists, such as the ideal amount of force to apply in each grip experiment. As a result, an early prototype to study the amount of force involved in these experiments was created. The hardware has wireless communication interacting with a mobile application. In the current state-of-the-art, there is a low cost architecture of hardware operation and production, making possible its use and adhesion in therapeutic studies with technological demand. To identify the grip strength, piezoresistive sensors as fingertip touch sensors were used. The hardware is equipped with capabilities to receive and store online data from each experiment, sending the results to the cloud and providing access to historical data. Experiments were performed to determine the viability and performance of each fingertip sensor. The results show that the smart glove can detect the grip strength and communicate the data without issues.
\end{abstract}

\section{Introduction}

In the movement of the human body, the transmission of each information is sent to the human limbs and the extensive network of neurons, in the form of a pulse signal, is created to generate action potentials to the requested muscle groups [Petersen et al. 2018]. Healthy habits contribute to the longevity of neural cells. However, factors such as age, chronic diseases, and drug use accelerate the process of degradation of the myelin sheath, protective lipoprotein layer, reducing the velocity of nerve impulses to target limbs [Mummadi et al. 2017]. As a result of this, the constant maintenance of this system is necessary to provide excellent motor coordination.

The failure of the neural commands is classified by the origin of the problem as: incoordination occurs when commands are sent to the target member and the response 
occurs in adjacent members, which is associated with chronic diseases. Dislocation occurs in the same way but originates from muscular injuries or lack of physical activity, common in the first years of life. During Daily Activity (DA), the work shows of palm muscles require repetition at different instants of time. Among them, energy conversion to the processes of gesture communication oriented typing and morphological manipulation. The quantification of force is different in each activity [Preetham et al. 2013].

To evaluate muscle quality, experiments are performed, that is, daily replication of testing on limbs. In functional biomechanical evaluations (BMFE), dynamometers are used to obtain palmar measurement data in patients. These types of equipment are responsible for quantitatively measuring the maximum isometric muscular strength of the hand, and its use is recommended [Tolvanen et al. 2017]. The BMFE is subdivided into various types of experiments and classifications of movements for different limbs using the dynamometers [Tolvanen et al. 2017]. However, the present study was devoted to three movements present in end-to-end palmar grip experiment (EEPG), being: tweezers, three-finger tweezers, and three-dimensional tweezers. For this type of experiment, it is essential to use the maximum contact fingers area with the piezoresistive sensors.

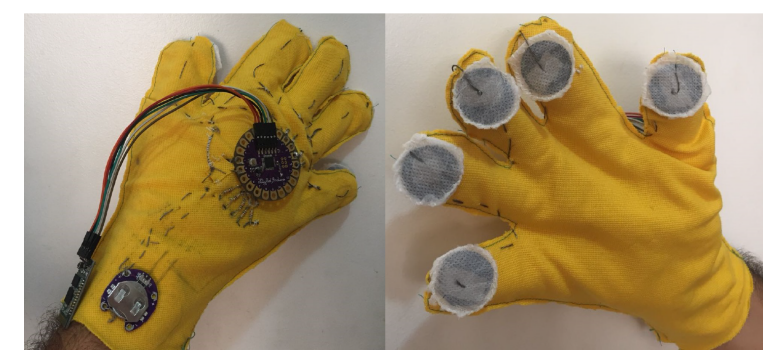

Figure 1. PHOTOGRAPHY OF A SMART GLOVE

The objective of this work is the proposal and deployment of a hardware (Fig.1) capable of performing palmar grip experiments to identify the different force applied levels in each experiment. The current work presents results of greater intensity of grip and progression over time. Thus, suggesting a detailed and feasible examination for the identification of possible pathologies associated with chronic diseases or muscular injuries.

The rest of this work is organized as follows. Section 2 elaborates on the background and motivation. Section 3 presents the basic principle of the Palmar Sensor and Section 4 its implementation. Results analysis and discussion are aavailable in Section 5 and Section 6 concludes the paper and suggests future works.

\section{Background and Motivation}

The current state-of-the-art represents a growing reality in research and investments in computing present in areas such as health and robotics [Sukenik et al. 2019]. In this scope, some papers use the Velostat, Semiconductor Polymer Composite, for the replacement of rigid and inflexible sensors. In the table, 1 are being compared works that performed the study or analysis of equipment that make a measurement equivalent to this work. Unlike the other single-point sensor models which consists of using the Velostat between two polarized electrodes, the authors [Roll and Hardison 2017] present longitudinal cuts of the Velostat forming a matrix. In this configuration, the piezoresistive sensors are capable of mapping a large area of the hand to obtain the applied force, thus making it 
possible to measure values between 1 and 500k Pa. In the results, the sensor array showed high sensitivity in different applications of forces. Therefore, this work going to use the Velostat between two polarized electrodes to measure the palmar grip of the patient using the glove during the experiment.

Table 1. COMPARATIVE TABLE OF THE STATE-OF-THE-ART.

\begin{tabular}{|c|c|c|c|}
\hline Work & $\begin{array}{c}\text { Sensors } \\
\text { Types }\end{array}$ & Circuits & $\begin{array}{c}\text { Use } \\
\text { mode }\end{array}$ \\
\hline This Work & $\begin{array}{c}\text { Single } \\
\text { point }\end{array}$ & $\begin{array}{l}\text { Tension } \\
\text { divider }\end{array}$ & $\begin{array}{l}\text { Palmar } \\
\text { strength }\end{array}$ \\
\hline [Baldoli et al. 2017] & Array & $\begin{array}{l}\text { Tension } \\
\text { divider }\end{array}$ & $\begin{array}{l}\text { Measure } \\
\text { strength }\end{array}$ \\
\hline [Lin and Seet 2015] & $\begin{array}{l}\text { Single } \\
\text { point }\end{array}$ & $\begin{array}{c}\text { Not } \\
\text { identified }\end{array}$ & $\begin{array}{l}\text { Measure } \\
\text { strength }\end{array}$ \\
\hline [Giovanelli and Farella 2016] & Array & $\begin{array}{l}\text { Tension } \\
\text { divider }\end{array}$ & $\begin{array}{l}\text { Measure } \\
\text { strength }\end{array}$ \\
\hline [Mummadi et al. 2017] & $\begin{array}{c}\text { Not } \\
\text { identified }\end{array}$ & $\begin{array}{l}\text { Tension } \\
\text { divider }\end{array}$ & $\begin{array}{l}\text { Gesture } \\
\text { recognition }\end{array}$ \\
\hline [Tolvanen et al. 2017] & $\begin{array}{l}\text { Single } \\
\text { point }\end{array}$ & $\begin{array}{l}\text { Tension } \\
\text { divider }\end{array}$ & $\begin{array}{l}\text { Measure } \\
\text { pressure }\end{array}$ \\
\hline [Tsai et al. 2017] & $\begin{array}{c}\text { Single } \\
\text { point }\end{array}$ & $\begin{array}{l}\text { Tension } \\
\text { divider }\end{array}$ & $\begin{array}{l}\text { Measure } \\
\text { strength }\end{array}$ \\
\hline
\end{tabular}

\section{Basic Principle of the Palmar Sensor}

The measurement of palmar sensors allows the determination of skeletal muscle tension, demonstrated in Fig 3. The method and device use the mechanism of muscle contraction to identify the amount of force exerted under the skin. During hand movement, skeletal muscle tension varies at different levels of contractile force. However, this work analyzes the static muscular tensions; that is, there is no movement of the target limb.

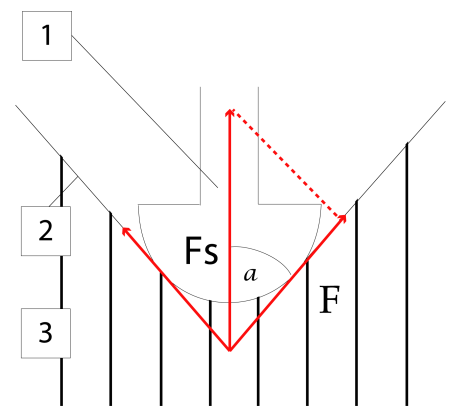

\section{Figure 2. REPRESENTATION MODEL SIMPLIFIED TO MEASURE PALMAR STRENGTH (1) SKIN CONTACT (2) PRIMARY AND SECONDARY CONNEC- TOR (3) PALMAR MENSURATION.}

The basic structure of the palmar sensor is organized in the sandwich arrangement with the Velostat between the conductors (Conductor - Velostat - Conductor). The sensor has been constructed in such a way that the skin is in contact with the primary conductor by impressing a pressure that makes contact with the secondary conductor. Measurement of muscle tension produces a force (Fs) in the longitudinal direction of the limb. The force exerted between the skin, and the primary conductor is transferred to the Velostat 
and the secondary conductor, thereby exciting a resistance variation, related in a manner proportional to the gripping force. Decomposing Fs:

$$
\mathrm{Fs}=\mathrm{F} \cos (\mathrm{a})
$$

Where a is the angle made with the surface, $\mathrm{F}$ and Fs represent the directions of each force. In practical situations, this Equation 1 disregards variables originating from external forces during skeletal muscle tension. However, this type of model is applied to simplify the behavior of the muscular layers and conductors of the transfer of forces. For force values, this paper was based on the work [Lin and Seet 2015]. Which uses a voltage divider to check the intensity at each layer hold level. Thus, this work uses the Equation 2 proposed by the [Ferreira et al. 2011], the viscoelastic model for Semiconductor Polymer Composite relating tension and force.

$$
F s=0.569 \log (44.98 V)
$$

\section{Implementation}

In this work was implemented wearable hardware, integrated into a network architecture. The implementation of the proposed prototype during EEPG experiments is divided into four layers: Components, Algorithm, Communication, and Testbed.

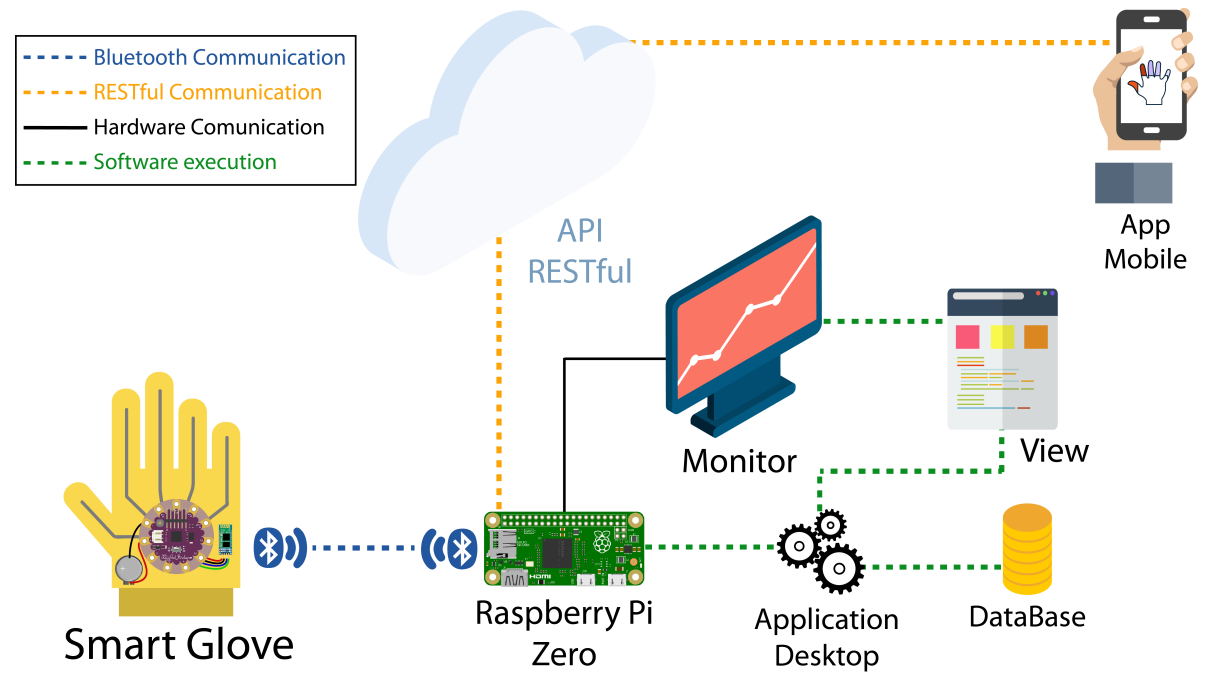

Figure 3. COMMUNICATION INFRASTRUCTURE PROTOTYPE ARCHITECTURE.

\subsection{Hardware}

The wearable computing was used to provide grip experiments. The main hardware components are shown diagrammatically in Fig. 3.

- Microcontroller: the Atmega168V connected between the palm sensor and the Bluetooth module was used. The analog signals are produced by the sensors when they are identified and interpreted by the microcontroller, after that step the voltage ranges on the sensor are identified, and the applied force values are encoded. 
- Bluetooth module: The selected module was the Bluetooth RN-41 class 1 with a serial transmission rate (RX/TX) of $115200 \mathrm{bps}$. The security planned the choice of this communication in transmitting and transmitting the data, and access in a range of electronic devices. Another reason was that when implementing the HC-05 and HC-06, others types of Bluetooth module, it was observed that there is a problem in the data transmission rate since its operation is of $8 \mathrm{MHz}$ and the microcontroller is of $16 \mathrm{MHz}$ for this reason the best model for this one application type is the RN-41.

\subsection{Software}

The software running on the microcontroller receives the analog data, arranging them by separating independently for each sensor. During the capture, the process of filtering of the analog signal is carried out using moving average filter to avoid noise and distortions in the force readings denoted by Equation 3. Then the intervals are converted to force values. After the process, the converted values are organized and sent via Bluetooth in packages composed of force values. It then passes on to the Application Programming Interface (API) in the cloud, for storage in the database, and to provide access to mobile applications. The received data contains the coded values corresponding to the five sensors, and at the beginning of each reading, an identifier is assigned to each finger.

$$
y(i)=\frac{1}{M} \sum_{j=0}^{M-1} x(i+j)
$$

When initializing, the software identifies the reading of the data by performing the mapping of the sensors pressed. During the mapping of the corresponding values by each sensor, the data is stored in the cloud and sent to the smartphone, where it is going to be displayed graphically. Before printing the data of each sensor, the organization will be done by location in hand. That is, each finger shows the intensity of the force applied by a color scale of cold and warm tones. At the end of each experiment, a line graph is generated to identify the force progression in Newton per second (N/s) for each movement performed.

\subsection{Communication}

The communication implementation using Bluetooth Low Energy (BLE), to obtain compatible access to smartphones. The security of the data sent between the hardware and the glove is also considered using authentication key pairing protocols. The BLE communication, developed by the Special Interest Group (SIG), offers a standard of energy efficiency that could be used in equipment with cellular batteries, including wearable. BLE has a suitable topology for health applications. Additionally, this technology operates with a $150 \mathrm{~m}$ range in the open field at a data rate with 1 megabyte per second (Mbps) band, low latency, and $2.4 \mathrm{GHz}$ band. From the data sent to the mobile application, the smartphone connected to the Internet accesses a cloud address and sends the data collected to a database, to provide access to the patient's history.

\subsection{Testbed}

For analyzes different body postures for the prototype to be used to establish a clinically relevant and reproducible methodology. The standardized posture is proposed by 
the American Society of Hand Therapists (ASHT). The experiments are performed with patients sitting in a chair with a straight upright back, without arm support, with elbow flexed at a 90-degree angle and forearm in neutral position. Another recommendation of the ASHT is the choice of the non-dominant hand, that is, with less grip strength. In most of the studies performed, the technique uses the ratio of three measurements, in one-minute intervals, to avoid muscle fatigue where the highest value measurement is assumed to result from [Baldoli et al. 2017]. Sex and age are the main determinants of MPF in healthy subjects [Annetta et al. 2019]. Thus, this paper followed the methodology recommended and standardized by ASHT, also present in the EEPG experiment. For the testbed, the exams are classified into types of patients, hold, and experiments.

For the patients, it was necessary to identify the sex, age, and history of lesions, since these variables affect the reading of the sensors for each experiment. Grip experiments were performed on men aged between 21 and 45 years, in a small sample space, with no history of neuropathies and muscular lesions.

\subsubsection{Gripping of strength and precision gripping experiments}

In this paper, the classic study promoted by [Fuller et al. 2019] was used. It is affirming that the human body presents two types of palmar grip: gripping of strength and precision gripping. Being precision gripping a delicate application on specific equipment (sensor), already the grip of force would be a hold of the total force impressed by the hand on this sensor. Also, each experiment was classified by the types of grip. Classified in: spherical grip, cylindrical grip, hook grip, and lateral grip. However, for this work, the experiments performed are spherical and cylindrical.

In the experiments, three movements were used in the EEPG experiments. Among them are: Tweezers, Tridigital Tweezers, and Global Pentadigital Tweezers. In the tweezers, the patient performs the spherical type precision grip experiment, however, there is an overlap of the fingers with the flexor muscle of the gauge and flexor muscle of the thumb (Fig. 4.a). In the Tridigital Tweezers, the patient performs a spherical type precision grip experiment with the flexor digitorum deep muscles, and thumb flexor muscle, that is, the union of the thumb, forefinger and middle finger against one object of small volume (Fig. 4.b). In the Pentadigital Global Tweezers, the spherical type force grip is performed, working the deep flexor muscles and flexor longus muscle of the thumb. However, with the aid of the superficial flexor muscles of the finger and the interosseous muscles, that is, the patient prints a force against the object and it returns with a resultant force of equal value for maintaining balance (Fig. 4.c).

\section{Results Analysis and Discussion}

The preliminary results obtained in this work showed the measurement of the amount of force applied to the sensors implemented and used in the EEPG experiments. Measurements were performed with the non-dominant hand of each patient, following the protocol described in the previous sections. Due to the lack of research using dynamometers in EEPG experiment, this paper proved the normality of the group of experiment with the supervised analysis, through the accompaniment of a physiotherapist. In this section, the behavior of the graphs during the performance of each experiment is presented, dividing 


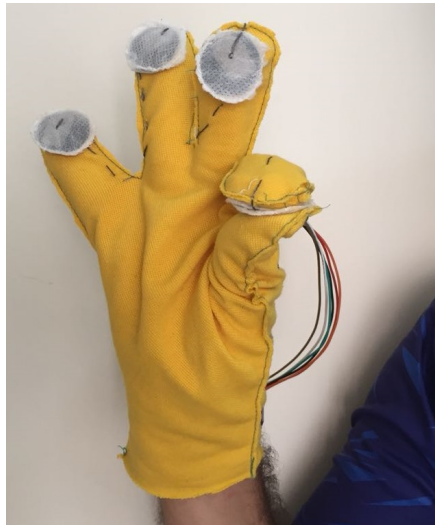

(a) TWEEZERS

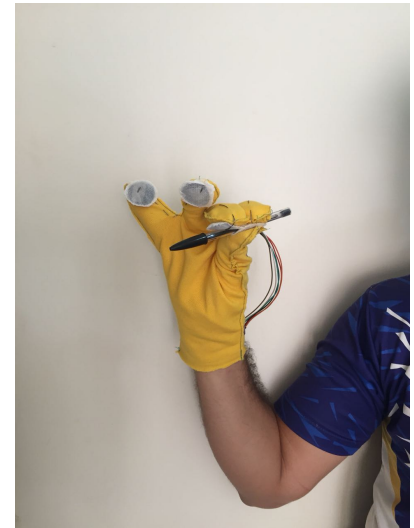

(b)
TRIDIG-

ITAL

TWEEZ-

ERS

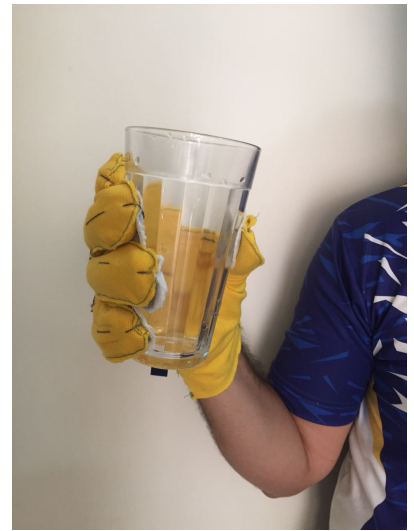

(c) PENTADIG-

ITAL

GLOBAL

PRE-

SEASON

Figure 4. GRIPPING OF STRENGTH AND PRECISION GRIPPING EXPERIMENTS.

into two types of graphs - the first, in tension for time and the second of force per time.

In the tweezers experiment shown in the graphs of Fig.5, it was observed that in the first second, patients print an accommodating force between the index fingers and thumbs. In the other four seconds, there was a low variation of the force values. With the stability of these fingers, normality was validated; that is, the motor coordination acceptable for this movement. In the tridigital tweezers experiment presented in the graphs of Fig. 6, the index fingers, and thumbs during the first seconds showed sharp variations in tension. However, for the force-time relationship, the intervals were close. Already for the middle finger, there were minimal variations of both strength and tension. In this experiment, the thumb was also observed as a support base and the index finger and thumb as an adder of forces that cancel with the force of sustentation that is, the maintenance of the static balance. The global pentadigital hold experiment presented in Fig. 7, shows different results of the other experiment during the first second, for both tension and force. In this experiment, the use of all the fingers of the hand offered a better distribution of the force to hold and control an object of high volume.

In the works discussed in section 2, different models of sensors were presented using Velostat to measure the amount of force, grip, and mapping of sign language. In the comparative table, other research related to Velostat was increased. Among the information presented, the proposed configuration for the circuits formed in a matrix and the single point was used the voltage divider. The use of this material is necessary for the significant part of the works present in the current state of the art, being used to create sensors of force or pretension. Also, the sensors manufactured from the SCP are adaptable to computing, replacing rigid and inflexible sensors.

In the results presented, it can be observed that although this material is widely marketed as an anti-static package, it is necessary to promote mechanical compression and tensile experiments to investigate the resistance behavior to high levels of grip and the breaking threshold when being drawn. In this work, wireless communication to connect 

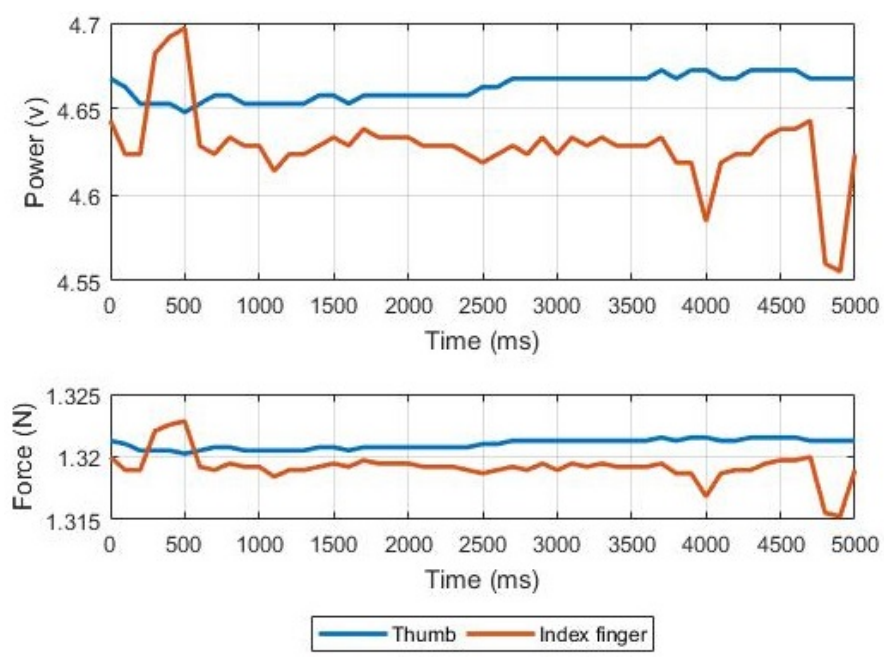

Figure 5. RESULTS OF PALMAR TWEEZERS GRIPPER EXPERIMENTS.
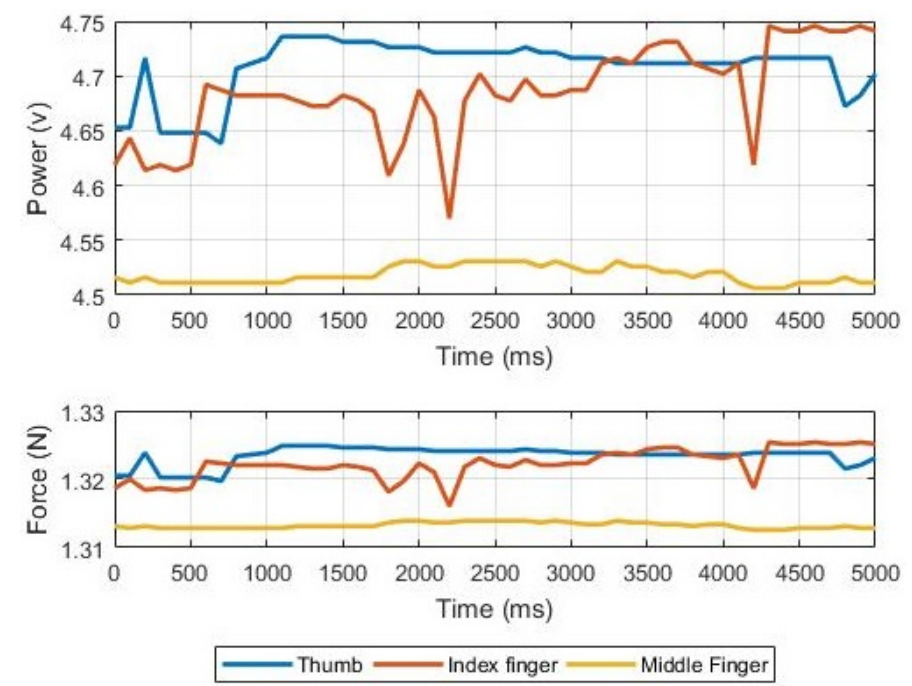

Figure 6. RESULTS OF PALMAR TRIGITAL TWEEZERS GRIPPER EXPERIMENTS.

a mobile application was used. However, the work is limited to the availability of results to the user. Different from this work, an interface that offers graphics resources and data access by the user is proposed.

The implementation along with its proposed architecture assisted the physiotherapists in the EEPG experiment to study the minimum, maximum strength and the progression imprinted over time. Another contribution was the comfort provided to the users during the experiments, thus generating results with lower chances of errors. With the mobile application, the results about the grip strength optimized the response time on possible in coordination during the experiments performed. In the analyzes collected by the glove, it was possible to feed one database for each patient, in order to provide printed results and to define standards for each study sample. 

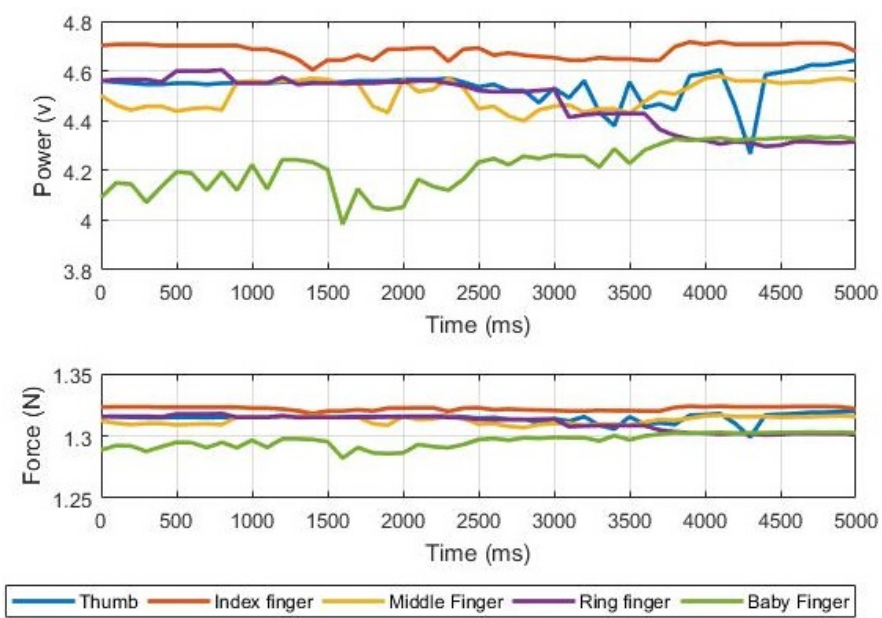

Figure 7. PALMAR PENTADIGITAL GLOBAL PRESEASON GRIPPER RESULTS.

\section{Conclusion and Future Work}

In this paper, hardware was modeled with wearable wear-sensitive palatal sensor technology, present at the ends of each finger. The objective of this work is to analyze and present the applied force on the sensors, the behavior of the force in healthy people and to return feedback of the minimum and maximum amounts applied in each experiment, by the physiotherapists. The overall system design is illustrated for the first time with the communication and software implementation, and the presence of wearable. In the software implementation, the signal treatment coming from the sensors and the commands made in the mobile application was carried out. The three types of end-to-end experiments were performed using the smart glove proposed and implemented in this work. Additionally, the experiments were performed based on palmar gripper analysis patterns present in the current state of the art. After the success of the implementations, the hardware was able to assist in the rehabilitation of patients with neuromuscular problems occasioned by the hand such as Guilan Barre Syndrome, and Accidents and among other patients.

As future work, the hardware and its communication architecture may have improvements to identify the force values with greater precision, so that it can promote experiments with an extensive sample space and with patients of diverse age groups. Additionally, it is intended to use the data generated by the experiments to perform machine training to provide an identification of patterns of normality about gender and age.

\section{Acknowledgments}

This work was partially supported by national funding from the FCT - Fundação para a Ciência e a Tecnologia through the UID/EEA/50008/2019 Project; by RNP, with resources from MCTIC, Grant No. 01250.075413/2018-04, under the Centro de Referência em Radiocomunicações - CRR project of the Instituto Nacional de Telecomunicações (Inatel), Brazil; and by Brazilian National Council for Research and Development (CNPq) via Grant No. 309335/2017-5.

\section{References}

Annetta, N. V., Friend, J., Schimmoeller, A., Buck, V. S., Friedenberg, D. A., Bouton, C. E., Bockbrader, M. A., Ganzer, P. D., Colachis IV, S. C., Zhang, M., et al. (2019). 
A high definition noninvasive neuromuscular electrical stimulation system for cortical control of combinatorial rotary hand movements in a human with tetraplegia. IEEE Transactions on Biomedical Engineering, 66(4):910-919.

Baldoli, I., Maselli, M., Cecchi, F., and Laschi, C. (2017). Development and characterization of a multilayer matrix textile sensor for interface pressure measurements. Smart Materials and Structures, 26(10):104011.

Ferreira, A. C. d. C., Shimano, A. C., Mazzer, N., Barbieri, C. H., Elui, V. M. C., and Fonseca, M. d. C. R. (2011). Grip and pinch strength in healthy children and adolescents. Acta Ortopédica Brasileira, 19(2):92-97.

Fuller, J., Cronin-Golomb, A., Gatchel, J., Norton, D., Guzmán-Vélez, E., Jacobs, H., Hanseeuw, B., Pardilla-Delgado, E., Artola, A., Baena, A., et al. (2019). Biological and cognitive markers of presenilin1 e280a autosomal dominant alzheimer's disease: A comprehensive review of the colombian kindred. The journal of prevention of Alzheimer's disease, 6(2):112-120.

Giovanelli, D. and Farella, E. (2016). Force sensing resistor and evaluation of technology for wearable body pressure sensing. Journal of Sensors, 2016.

Lin, X. and Seet, B.-C. (2015). A linear wide-range textile pressure sensor integrally embedded in regular fabric. IEEE Sensors Journal, 15(10):5384-5385.

Mummadi, C. K., Leo, F. P. P., Verma, K. D., Kasireddy, S., Scholl, P. M., and Van Laerhoven, K. (2017). Real-time embedded recognition of sign language alphabet fingerspelling in an imu-based glove. In Proceedings of the 4th international Workshop on Sensor-based Activity Recognition and Interaction, page 11. ACM.

Petersen, R. C., Lopez, O., Armstrong, M. J., Getchius, T. S., Ganguli, M., Gloss, D., Gronseth, G. S., Marson, D., Pringsheim, T., Day, G. S., et al. (2018). Practice guideline update summary: Mild cognitive impairment: Report of the guideline development, dissemination, and implementation subcommittee of the american academy of neurology. Neurology, 90(3):126-135.

Preetham, C., Ramakrishnan, G., Kumar, S., Tamse, A., and Krishnapura, N. (2013). Hand talk-implementation of a gesture recognizing glove. In 2013 Texas Instruments India Educators' Conference, pages 328-331. IEEE.

Roll, S. C. and Hardison, M. E. (2017). Effectiveness of occupational therapy interventions for adults with musculoskeletal conditions of the forearm, wrist, and hand: a systematic review. American Journal of Occupational Therapy, 71(1):1-12.

Sukenik, A., Rücker, J., and Maldener, I. (2019). Dormant cells (akinetes) of filamentous cyanobacteria demonstrate a great variability in morphology, physiology, and ecological function. In Cyanobacteria, pages 65-77. Elsevier.

Tolvanen, J., Hannu, J., and Jantunen, H. (2017). Hybrid foam pressure sensor utilizing piezoresistive and capacitive sensing mechanisms. IEEE Sensors Journal, 17(15):4735-4746.

Tsai, Y.-C., Ma, C.-W., Lin, Y.-H., and Yang, Y.-J. (2017). Development of a large-area $8 \times 8$ tactile sensing array with high sensitivity. Sensors and Materials, 29(3):303-309. 